\title{
ROBUST POLE PLACEMENT IN A LMI REGION FOR TAKAGI-SUGENO MODELS: APPLICATION TO VEHICLE SPACING CONTROL ${ }^{1}$
}

\author{
S. Delprat ${ }^{1}$, P.F. Toulotte ${ }^{1 \& 2}$, T.M. Guerra ${ }^{1}$, J. Boonaert ${ }^{2}$ \\ ${ }^{1}$ LAMIH UMR CNRS 8530, University of Valenciennes et du Hainaut-Cambrésis, \\ Le Mont Houy, 59313 Valenciennes CEDEX 9 France \\ 2 SID, Ecole des Mines de Douai, 764 bvd Lahure, 59508 Douai, France \\ E-Mail : \{toulotte, boonaert\}@ensm-douai.fr, \{guerra, delprat\}@univ-valenciennes.fr
}

\begin{abstract}
Robust pole placement in a LMI region for Takagi-Sugeno (Takagi \& Sugeno 1985) fuzzy models is presented. The objective is to find a set of Linear Matrix Inequalities in order to ensure that the linear models poles remain in a specified region of the complex plane, even in presence of model uncertainties. As an illustration, the obtained conditions are applied to the spacing policy control of an automated electric vehicle. Simulation and real time results are presented. Copyright (C) 2005 IFAC
\end{abstract}

Keywords: Fuzzy control, Non linear control, Robustness, Vehicle control.

\section{INTRODUCTION}

Takagi Sugeno (TS) models (Takagi \& Sugeno 1985) allow representing exactly a large class of non linear systems in a compact set of the state vector. They consist of a collection of linear models blended by non linear functions. They have been widely used for control of non linear systems: stabilization (Guerra \& al. 2003), (Tanaka \& al. 1998), with bounded uncertainties (Lauber \& al. 2004), (Tong \& al. 2002) and so on. One way to reach some performances was also investigated through pole placement in a Linear Matrix Inequality (LMI) region for each linear models (Hong \& Langari 2000).

Note that a pole placement for each linear models of a TS model in a LMI region does not allow specifying exactly the dynamics of the non linear model in closed loop. Nevertheless it is still a very interesting way of tuning the close loop dynamics. In this paper we present a control scheme in order to ensure that the poles of the linear models remain in a LMI region even in presence of bounded uncertainties.

The first part of this paper presents some new LMI conditions based on a Lyapunov quadratic function and model poles placement for uncertain systems. The second part describes the spacing control of an automated vehicle.

\section{ROBUST CONTROL WITH POLES PLACEMENT IN A LMI REGION}

We consider the following TS uncertain model (Taniguchi \& al. 2001):

$\left\{\begin{array}{l}\dot{x}(t)=\sum_{i=1}^{n} h_{i}(t)\left(\left(A_{i}+\Delta A_{i}\right) x(t)+\left(B_{i}+\Delta B_{i}\right) u(t)\right) \\ y(t)=\sum_{i=1}^{n} h_{i}(t)\left(C_{i}+\Delta C_{i}\right) x(t)\end{array}\right.$

with $\sum_{i=1}^{n} h_{i}(t)=1$ and $h_{i}(t) \geq 0$

For sake of convenience, the following notations are used when there is no ambiguity:

$$
\begin{aligned}
& Y_{h}=\sum_{i=1}^{r} h_{i}(z(t)) Y_{i}, \\
& \Sigma^{+}(A)=A+A^{T}, \Sigma^{-}(A)=A^{T}-A
\end{aligned}
$$

Model (1) can be written into a more compact form:

$\left\{\begin{array}{l}\dot{x}(t)=\left(A_{h}+\Delta A_{h}\right) x(t)+\left(B_{h}+\Delta B_{h}\right) u(t) \\ y(t)=\left(C_{h}+\Delta C_{h}\right) x(t)\end{array}\right.$

We consider the following Parallel Distributed Compensation (PDC) control law (Wang \& al. 1996): $u(t)=-F_{h} \cdot x(t)$

Yields the closed loop:

\footnotetext{
${ }^{1}$ This work was granted in part by the Region Nord Pas de Calais and the FEDER (European funds) under grant of the AUTORIS project held by the GRAISyHM. (AUTORIS: AUTOmatique pour la Route Intelligente et Sûre. GRAISyHM: Regional Research Group in Automation and Man Machine Systems).
} 


$$
\left\{\begin{array}{l}
\dot{x}(t)=\left(A_{h}-B_{h} F_{h}\right) x(t)+\left(\Delta A_{h}-\Delta B_{h} F_{h}\right) x(t) \\
y(t)=\left(C_{h}+\Delta C_{h}\right) x(t)
\end{array}\right.
$$

A classical problem is then to find the control gains $F_{i}$ that ensure the closed loop stability of the uncertain model (Taniguchi \& al. 2001) but the so obtained control gains may lead to poor dynamic performances.

For models without uncertainties, pole placement in LMI region was studied in the case of linear systems (Chilali \& al. 1999) and for TS fuzzy models (Hong \& Langari 2000). Of course specifying the closed loop dynamics of each linear model will not ensure a property to the TS model as the nonlinear scalar membership functions $h_{i}(t)$ are not taken into account. However, it is still a very interesting way of tuning the closed loop, for example it may be used to restrict the controller dynamics and to achieve an expected transient behavior (Hong \& Langari 2000). In this paper we propose to address the following problem: a LMI region being defined is there any PDC law ensuring that each linear model poles lay in this region, even in presence of bounded uncertainties?

We define the region $\mathfrak{R}$, fig. 1 , according to three parameters $\lambda, \rho$ and $\vartheta$. These parameters allow defining the transient response dynamics and the damping factor. The eigenvalues of a matrix $A$ lay in the LMI region $\mathfrak{R}$ of the complex plane if there exists a common matrix $P=P^{T}>0$ such that (Chilali \& al. 1999):

$A^{T} P+P A+2 \cdot \lambda \cdot P<0$

$\left[\begin{array}{cc}-\rho P & A^{T} P \\ P A & -\rho P\end{array}\right]<0$

$\left[\begin{array}{cc}\text { с } \vartheta\left(A^{T} P+P A\right) & s \vartheta\left(-A^{T} P+P A\right) \\ s \vartheta\left(A^{T} P-P A\right) & \text { с } \vartheta\left(A^{T} P+P A\right)\end{array}\right]<0$

$P>0$

with: $s \vartheta=\sin (\vartheta)$ and $c \vartheta=\cos (\vartheta)$.

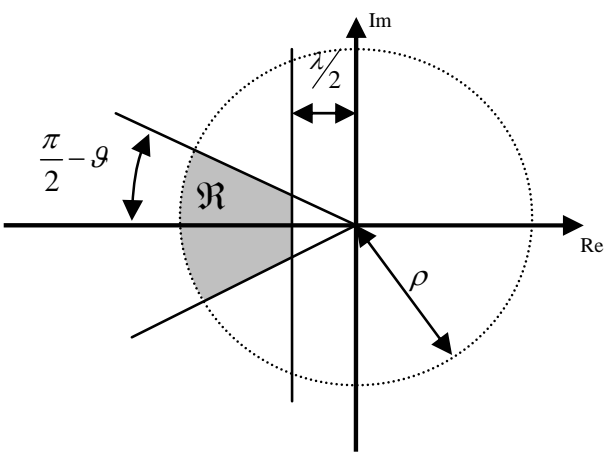

Fig. 1: Considered LMI region

As a minimum requirement, we want to ensure the closed loop stability by the mean of a candidate quadratic Lyapunov function.

$$
\begin{aligned}
& V(x(t))=x(t)^{T} P x(t) \\
& P>0
\end{aligned}
$$

Moreover we also want to constraint the linear models poles of the TS model in the half plane defined by $\operatorname{Re}(x)<-\frac{\lambda}{2}, x \in \mathbb{C}$, so a decay rate $\lambda>0$ is used.

$\dot{V}(x(t)) \leq-\lambda V(x(t))$

With $X=P^{-1}$, (9) can be written:

$$
\begin{aligned}
& \Sigma^{+}\left(X A_{h}{ }^{T}-M_{h}{ }^{T} B_{h}{ }^{T}\right)+ \\
& \Sigma^{+}\left(X \Delta A_{h}{ }^{T}-M_{h}{ }^{T} \Delta B_{h}{ }^{T}\right)+\lambda X<0
\end{aligned}
$$

The bounded uncertainties are specified in an usual way: $\quad \Delta A_{h}=H a_{h} \Delta a_{h}(t) E a_{h}, \quad \Delta B_{h}=H b_{h} \Delta b_{h}(t) E b_{h}$ using constant matrices $H a_{h}, H b_{h}, E a_{h}, E b_{h}$ and uncertain bounded terms $\Delta a_{h}(t)$ and $\Delta b_{h}(t)$ such that (Taniguchi \& al. 2001) (Lauber \& al. 2004):

$\Delta a_{h}^{T}(t) \Delta a_{h}(t) \leq I, \Delta b_{h}{ }^{T}(t) \Delta b_{h}(t) \leq I$

Let us recall some classical properties.

Property $n^{\circ} 1$ (Xie \& De Souza 1992): with $X, Y$, $S>0$ matrices of appropriate dimension:

$X^{T} Y+Y^{T} X \leq X^{T} S X+Y^{T} S^{-1} Y$

Property n²: Schur’s complement

$Y+X^{T} R X<0 \Leftrightarrow\left[\begin{array}{cc}Y & X^{T} \\ X & -R^{-1}\end{array}\right]<0 \quad R>0$

Using properties 1 and 2 and (11) on inequality (10) leads to:

$\left[\begin{array}{ccc}Y & (*) & (*) \\ E a_{h} X^{T} & -\tau a_{h h} I & 0 \\ E b_{h} M_{h} & 0 & -\tau b_{h h} I\end{array}\right]<0$

with:

$$
\begin{aligned}
Y= & \Sigma^{+}\left(X A_{h}{ }^{T}-M_{h}{ }^{T} B_{h}{ }^{T}\right) \\
& +\tau a_{h h} H a_{h} H a_{h}{ }^{T}+\tau b_{h h} H b_{h} H b_{h}{ }^{T}+\lambda X
\end{aligned}
$$

Using (6) on the closed loop (4), we obtain:

$$
\begin{aligned}
& {\left[\begin{array}{cc}
-\rho X & (*) \\
A_{h} X-B_{h} M_{h} & -\rho X
\end{array}\right]+\Sigma^{+}\left(\left[\begin{array}{c}
0 \\
H a_{h} \Delta a_{h}
\end{array}\right]\left[\begin{array}{ll}
E a_{h} X & 0
\end{array}\right]\right)+} \\
& \Sigma^{+}\left(\left[\begin{array}{c}
0 \\
H b_{h} \Delta b_{h}
\end{array}\right]\left[\begin{array}{ll}
-E b_{h} M_{h} & 0
\end{array}\right]\right)<0
\end{aligned}
$$

Then using properties 1 and 2 twice, (16) is satisfied if:

$$
\left[\begin{array}{cccc}
-\rho X & (*) & (*) & (*) \\
A_{h} X-B_{h} M_{h} & Y^{\prime} & 0 & 0 \\
E a_{h} X & 0 & -\tau a_{h h}{ }^{\prime} I & 0 \\
E b_{h} M_{h} & 0 & 0 & -\tau b_{h h}{ }^{\prime} I
\end{array}\right]<0
$$

With $Y^{\prime}=-\rho X+\tau a_{h h}{ }^{\prime} H a_{h} H a_{h}{ }^{T}+\tau b_{h h}{ }^{\prime} H b_{h} H b_{h}{ }^{T}$

Using (7) on the closed loop (4) leads to:

$$
\begin{aligned}
& {\left[\begin{array}{ll}
\Phi_{11} & \Phi_{21}{ }^{T} \\
\Phi_{21} & \Phi_{11}
\end{array}\right]+\Phi_{2}+\Phi_{3}<0} \\
& \Phi_{11}=c \vartheta \cdot \Sigma^{+}\left(\left(A_{h}-B_{h} L_{h}\right) X\right) \\
& \Phi_{21}=s \vartheta \cdot \Sigma^{-}\left(\left(A_{h}-B_{h} L_{h}\right) X\right)
\end{aligned}
$$


$\Phi_{2}=\Sigma^{+}\left(\left[\begin{array}{cc}H a_{h} \Delta a_{h} & 0 \\ 0 & H a_{h} \Delta a_{h}\end{array}\right]\left[\begin{array}{cc}c \vartheta \cdot E a_{h} X & s \vartheta \cdot E a_{h} X \\ -s \vartheta \cdot E a_{h} X & \text { с } \cdot E a_{h} X\end{array}\right]\right)$

$\Phi_{3}=\Sigma^{+}\left(\left[\begin{array}{cc}H b_{h} \Delta b_{h} & 0 \\ 0 & H b_{h} \Delta b_{h}\end{array}\right]\left[\begin{array}{cc}-c \vartheta \cdot E b_{h} M_{h} & -s \vartheta \cdot E b_{h} M_{h} \\ s \vartheta \cdot E b_{h} M_{h} & -c \vartheta \cdot E b_{h} M_{h}\end{array}\right]\right)$

Using properties 1 and 2 on (18), we obtain:

$\left[\begin{array}{cccccc}Y_{11}^{\prime \prime} & \left(^{*}\right) & \left(^{*}\right) & \left(^{*}\right) & 0 & 0 \\ E a_{h} X & -\tau a_{h h}{ }^{\prime \prime} I & 0 & 0 & 0 & 0 \\ E b_{h} M_{h} & 0 & -\tau b_{h h}{ }^{\prime \prime} I & 0 & 0 & 0 \\ Y_{41}^{\prime \prime} & 0 & 0 & Y_{11}^{\prime \prime} & \left(^{*}\right) & \left(^{*}\right) \\ 0 & 0 & 0 & E a_{h} X & -\tau a_{h h}{ }^{\prime \prime} I & 0 \\ 0 & 0 & 0 & E b_{h} M_{h} & 0 & -\tau b_{h h}{ }^{\prime \prime} I\end{array}\right]<0$

with:

$$
\begin{aligned}
Y_{11}^{\prime \prime}=\mathrm{c} \vartheta \cdot \Sigma^{+}\left(X A_{h}{ }^{T}-M_{h}{ }^{T} B_{h}\right) & \\
& +\tau a_{h h}{ }^{\prime \prime} H a_{h} H a_{h}{ }^{T}+\tau b_{h h}{ }^{\prime \prime} H b_{h} H b_{h}{ }^{T} \\
Y_{41}^{\prime \prime}= & \text { } \vartheta \cdot \Sigma^{-}\left(A_{h} X+B_{h} M_{h}\right) .
\end{aligned}
$$

Let us define from (14), (17) and (19).

$$
\Upsilon_{i j}^{1}=\left[\begin{array}{ccc}
Y_{i j}^{1} & (*) & (*) \\
E a_{i} X^{T} & -\tau a_{i j} I & 0 \\
E b_{i} M_{j} & 0 & -\tau b_{i j} I
\end{array}\right],
$$

with: $Y_{i j}^{1}=\Sigma^{+}\left(X A_{i}^{T}-M_{j}^{T} B_{i}^{T}\right)$

$$
\Upsilon_{i j}^{2}=\left[\begin{array}{cccc}
+\tau a_{i j} H a H a^{T}+\tau b_{i j} H b H b^{T}+\lambda X \\
-\rho X & (*) & (*) & (*) \\
A_{i} X-B_{i} M_{j} & Y_{i j}^{2} & 0 & 0 \\
E a_{i} X & 0 & -\tau a_{i j}{ }^{\prime} I & 0 \\
E b_{i} M_{j} & 0 & 0 & -\tau b_{i j}{ }^{\prime} I
\end{array}\right]
$$

with: $Y_{i j}^{2}=-\rho X+\tau a_{i j}{ }^{\prime} H a H a^{T}+\tau b_{i j}{ }^{\prime} H b H b^{T}$

$$
\Upsilon_{i j}^{3}=\left[\begin{array}{cc}
Y_{i j}^{3} & (*) \\
\Gamma_{i j}^{3} & Y_{i j}^{3}
\end{array}\right] \text {. }
$$

with: $\Gamma_{i j}^{3}=\left[\begin{array}{ccc}s \vartheta \cdot \Sigma^{-}\left(A_{i} X+B_{i} M_{j}\right) & 0 & 0 \\ 0 & 0 & 0 \\ 0 & 0 & 0\end{array}\right]$ and:

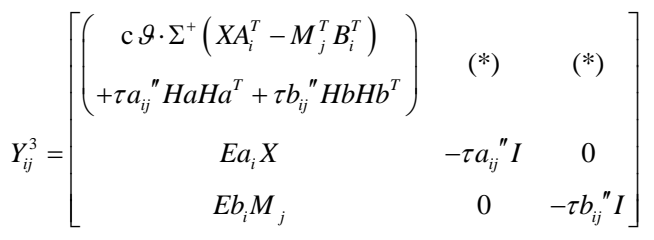

Let us note that the expressions used in (20), (21) and (22) suppose that $H a_{h}$ and $H b_{h}$ are constant over $h$. On the contrary, a simple Schur's complement must be applied on each term to ensure the equivalence and a LMI description.

Theorem 1: The closed loop model (4) is stable and all the linear models closed loop poles are located in a specified region $\mathfrak{R}$ if there exists matrices: $X=X^{T}>0, \quad M_{i}, \quad Q_{i i}^{k}>0, \quad \tau a_{i j}>0, \tau b_{i j}>0$, $\tau a_{i j}{ }^{\prime}>0, \quad \tau b_{i j}{ }^{\prime}>0, \quad \tau a_{i j}{ }^{\prime \prime}>0, \quad \tau b_{i j}{ }^{\prime \prime}>0$ and $Q_{i j}^{k}=\left(Q_{j i}^{k}\right)^{T}$ such that:
$\Upsilon_{i i}^{k}+Q_{i i}^{k}<0$

$\Upsilon_{i j}^{k}+\Upsilon_{j i}^{k}+Q_{i j}^{k}+Q_{j i}^{k}<0$

$Q^{k}=\left[\begin{array}{cccc}Q_{11}^{k} & Q_{12}^{k} & \cdots & Q_{1 r}^{k} \\ Q_{21}^{k} & Q_{22}^{k} & & \\ \vdots & & \ddots & \vdots \\ Q_{r 1}^{k} & & \cdots & Q_{r r}^{k}\end{array}\right]>0$

$i, j \in\{1, \ldots, r\}, j>i$ and $k \in\{1,2,3\}$.

Remark 3: The matrices $Q_{i i}^{k}>0$ and $Q_{i j}^{k}=\left(Q_{j i}^{k}\right)^{T}$ correspond to a relaxation scheme due to (Liu \& Zhang 2003).

\section{APPLICATION TO VEHICLE SPACING CONTROL}

The considered application is the longitudinal fuzzy control law for an electric car which is part of the virtual link project (AVIVA) included in the AUTORIS $^{2}$ project and held by the GRAISyHM ${ }^{3}$.

The main objective of the AVIVA project is to control the vehicle in order to maintain a defined distance with respect to the leading vehicle, Fig. 1. The vehicle's linear velocity is measured and the distance from the leader is estimated using stereovision sensor (Zayed \& al. 2003).

Several control laws have been proposed for this problem: a PID controller with gain scheduling is used in (Ioannou 1994), a second order sliding mode controller is used in (Nouvelière 2002), a fuzzysliding mode in (Lee 2002), etc.

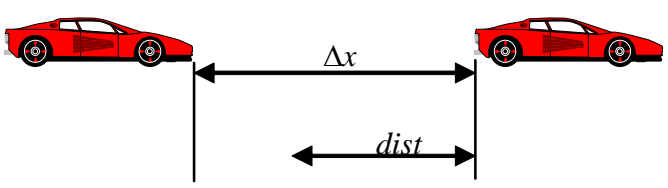

dist : distance to be reached $\Delta x$ : actual distance

Fig. 2: System configuration

2.1 Vehicle modeling and control

We consider the classical longitudinal bicycle model of the vehicle (Nouvelière 2002):

$$
\begin{aligned}
& \left(\frac{\left(M h^{2}+J_{r}+J_{f}\right) R_{g}^{2}+J_{e}}{h}\right) \ddot{x}(t)=T_{e}(t)- \\
& \quad R_{g}\left(T_{b}(t)+M_{r r}+h F_{a}(t)+M g h \sin (\Psi(t))\right) \\
& \text { with } F_{a}(t)=a_{x} \dot{x}(t)+c_{x} \dot{x}(t)^{2}
\end{aligned}
$$

In the AVIVA project, the considered vehicle is purely electric, so the gear ratio $R_{g}$ is constant. We will assume that the road surface is horizontal $\Psi(t)=0$ and that the necessary braking torque is provided by the motor. Yields the vehicle model:

\footnotetext{
2 AUTORIS: AUTOmatique pour la Route Intelligente et Sûre

3 GRAISyHM: Regional Research Group in Automation and Man Machine Systems
} 


$$
\begin{aligned}
& \left(\frac{\left(M h^{2}+J_{r}+J_{f}\right) R_{g}{ }^{2}+J_{e}}{h R_{g}}\right) \ddot{x}(t)= \\
& T_{e}(t)-R_{g}\left(M_{r r}+h a_{x} \dot{x}(t)+h c_{x} \dot{x}(t)^{2}\right)
\end{aligned}
$$

Defining $\quad C_{e}(t)=T_{e}(t)-R_{g} M_{r r}, \quad a=R_{g} h a_{x}$, $b=R_{g} h c_{x} \quad$ and $\alpha=\frac{\left(M h^{2}+J_{r}+J_{f}\right) R_{g}^{2}+J_{e}}{h R_{g}}$, the equation (28) becomes:

$$
\alpha \ddot{x}(t)=C_{e}(t)-a \dot{x}(t)-b \dot{x}(t)^{2}
$$

\begin{tabular}{|c|c|}
\hline$M$ & Mass of the vehicle (kg) \\
\hline$h$ & Height of the center of the wheel (m) \\
\hline$J_{r}$ & Rear wheel inertias (kg.m²) \\
\hline$J_{f}$ & Front wheel inertias (kg.m²) \\
\hline$R_{g}$ & Gear ratio \\
\hline$J_{e}$ & Engine / transmission inertias (kg.m²) \\
\hline$M_{r r}$ & Rolling resistance torque (N.m) \\
\hline$\Psi(t)$ & Road slope angle (deg) \\
\hline$T_{b}(t)$ & Brake torque (N.m) \\
\hline$T_{e}(t)$ & Engine torque (N.m) \\
\hline$\ddot{x}(t)$ & Longitudinal acceleration of the vehicle $\left(\mathrm{m} / \mathrm{s}^{2}\right)$ \\
\hline$\dot{x}(t)$ & Linear longitudinal speed of the vehicle $(\mathrm{m} / \mathrm{s})$ \\
\hline$F_{a}(t)$ & $\begin{array}{l}\text { Aerodynamic drag }(\mathrm{N}) \text {, related to the center of } \\
\text { the wheel }\end{array}$ \\
\hline
\end{tabular}

Table 1: Notations

At last, we assume that the motor has a first order dynamics on the torque, with a time constant $\tau$ :

$$
\dot{C}_{e}(t)=\frac{1}{\tau}\left(-C_{e}(t)+u(t)\right)
$$

where $u(t)$ is the torque setting point.

The objective is then to ensure that the distance between the leader vehicle and the following vehicle remains close to a desired distance:

$$
\operatorname{dist}(t)=d+e \dot{x}(t)+f \dot{x}(t)^{2}
$$

(29), (30) and (31) can be written into the form of a state space model:

$$
\left\{\begin{array}{l}
\dot{\bar{x}}(t)=\left[\begin{array}{cc}
\frac{-(a+b \dot{x}(t))}{\alpha} & \frac{1}{\alpha} \\
0 & -\frac{1}{\tau}
\end{array}\right] \bar{x}(t)+\left[\begin{array}{l}
0 \\
\frac{1}{\tau}
\end{array}\right] u(t) \\
y(t)=\left[\begin{array}{ll}
e+f \dot{x}(t) & 0] \bar{x}(t)
\end{array}\right.
\end{array}\right.
$$

with the state vector $\bar{x}(t)=\left[\begin{array}{ll}\dot{x}(t) & C e(t)\end{array}\right]^{T}$

The control objective is then to ensure that the output $y$ remains close to $\Delta x-d$.

The model (32) can be written as a Takagi-Sugeno model using the sector nonlinearity property (Tanaka $\&$ al. 1998) (Taniguchi \& al. 2001). In our case, the non-linearity involves the vehicle speed $\dot{x}(t)$, which is a bounded variable $\dot{x}(t) \in[0, \bar{v}]$ and the obtained
TS model (33) will exactly represents the nonlinear model (32).

$$
\left\{\begin{array}{l}
\dot{\bar{x}}(t)=\sum_{i=1}^{n} h_{i}(t)\left(A_{i}^{\prime} \bar{x}(t)+B_{i}^{\prime} u(t)\right) \\
y(t)=\sum_{i=1}^{n} h_{i}(t) C_{i}^{\prime} \bar{x}(t)
\end{array}\right.
$$

with $h_{1}(t)=\frac{\bar{v}-\dot{x}(t)}{\bar{v}}, h_{2}(t)=\frac{\dot{x}(t)}{\bar{v}}, B_{i}^{\prime}=B^{\prime}=\left[\begin{array}{l}0 \\ \frac{1}{\tau}\end{array}\right]$ $A_{1}^{\prime}=\left[\begin{array}{cc}-\frac{a}{\alpha} & \frac{1}{\alpha} \\ 0 & -\frac{1}{\tau}\end{array}\right], \quad A_{2}^{\prime}=\left[\begin{array}{cc}-\frac{(a+b \bar{v})}{\alpha} & \frac{1}{\alpha} \\ 0 & -\frac{1}{\tau}\end{array}\right]$, $C_{1}^{\prime}=\left[\begin{array}{ll}e & 0\end{array}\right]$ and $C_{2}^{\prime}=\left[\begin{array}{ll}e+f \bar{v} & 0\end{array}\right]$

The considered control law must ensure stability but also rejection of slow varying disturbances (road slope, etc.), therefore a control law with integral action is considered (Guerra \& Vermeiren 2003):

$$
u=\left[\begin{array}{ll}
-F_{h}^{\prime} & M_{h}
\end{array}\right]\left[\begin{array}{c}
\bar{x} \\
x_{I}
\end{array}\right]=F_{h}\left[\begin{array}{c}
\bar{x} \\
x_{I}
\end{array}\right]
$$

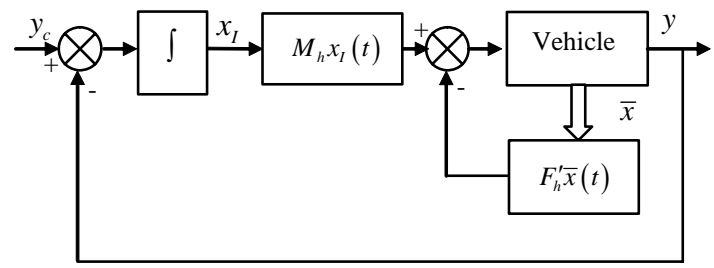

Fig. 3: control law with integral action

Let $\dot{x}_{I}=y_{c}(t)-y(t)$, the system can be written as:

$\left\{\begin{array}{l}\dot{\bar{X}}(t)=A_{h} \bar{X}(t)+B_{h} u(t)+\left(\begin{array}{l}0 \\ I\end{array}\right) y_{c}(t) \\ y(t)=C_{h} \bar{X}(t)\end{array}\right.$

where: $\bar{X}=\left[\begin{array}{c}\bar{X} \\ x_{I}\end{array}\right], \quad A_{h}=\left[\begin{array}{cc}A_{h}^{\prime} & 0 \\ -C_{h}^{\prime} & 0\end{array}\right], \quad B=B_{h}=\left[\begin{array}{c}B^{\prime} \\ 0\end{array}\right]$, and $C_{h}=\left[\begin{array}{ll}C_{h}^{\prime} & 0\end{array}\right]$.

Considering the longitudinal model (32) with uncertainties on the parameters $\alpha, a$ and $b$, and the control law (34), the following uncertain model is obtained:

$A_{1}=\left[\begin{array}{ccc}-\frac{a}{\alpha} & \frac{1}{\alpha} & 0 \\ 0 & -\frac{1}{\tau} & 0 \\ -e & 0 & 0\end{array}\right], \quad A_{2}=\left[\begin{array}{ccc}\frac{-a-b \bar{v}}{\alpha} & \frac{1}{\alpha} & 0 \\ 0 & -\frac{1}{\tau} & 0 \\ -e-f \bar{v} & 0 & 0\end{array}\right]$,

$B_{1}=B_{2}=B=\left[\begin{array}{l}0 \\ \frac{1}{\tau} \\ 0\end{array}\right], \quad \Delta A_{1}=\left[\begin{array}{ccc}-\Delta_{2} & \Delta_{1} & 0 \\ 0 & 0 & 0 \\ 0 & 0 & 0\end{array}\right]$,

$\Delta A_{2}=\left[\begin{array}{ccc}-\Delta_{2}-\Delta_{3} \bar{v} & \Delta_{1} & 0 \\ 0 & 0 & 0 \\ 0 & 0 & 0\end{array}\right], \quad \begin{aligned} & C_{1}=\left[\begin{array}{lll}e & 0 & 0\end{array}\right] \\ & C_{2}=\left[\begin{array}{lll}e+f \bar{v} & 0 & 0\end{array}\right]\end{aligned}$

$\Delta C_{1}=\Delta C_{2}=0$ and: $\Delta B=0$. 
Defining $\Delta_{1}=\bar{\Delta}_{1} \cdot \Delta v_{1}(t), \quad \Delta_{2}=\bar{\Delta}_{2} \cdot \Delta v_{2}(t)$ and $\Delta_{3}=\bar{\Delta}_{3} \cdot \Delta v_{3}(t) \quad$ such that $\quad \Delta v_{i}(t) \in[-1,1]$, $i \in\{1,2,3\}$, then:

$H a_{1}=H a_{2}=\left[\begin{array}{lll}1 & 1 & 1 \\ 0 & 0 & 0 \\ 0 & 0 & 0\end{array}\right]$,

$\Delta a_{1}(t)=\Delta a_{2}(t)=\left[\begin{array}{ccc}\Delta v_{1}(t) & 0 & 0 \\ 0 & \Delta v_{2}(t) & 0 \\ 0 & 0 & \Delta v_{3}(t)\end{array}\right]$,

$E a_{1}=\left[\begin{array}{ccc}0 & \bar{\Delta}_{1} & 0 \\ -\bar{\Delta}_{2} & 0 & 0 \\ 0 & 0 & 0\end{array}\right]$ and $E a_{2}=\left[\begin{array}{ccc}0 & \bar{\Delta}_{1} & 0 \\ -\bar{\Delta}_{2} & 0 & 0 \\ -\bar{\Delta}_{3} \bar{v} & 0 & 0\end{array}\right]$.

The vehicles parameters are given table 2 . The LMI region $\mathfrak{R}$ was defined by $\lambda=5, \rho=50$ and $\vartheta=2.35 \mathrm{rd}$. Considering the following uncertainties bounds $\quad \bar{\Delta}_{1}=7 e^{-5}, \quad \bar{\Delta}_{2}=1 e^{-3}, \quad \bar{\Delta}_{3}=4 e^{-3} \quad$ and parameter values defined in table 2 , some possible linear models poles locations of the TS model are shown figure 4 .

\begin{tabular}{|l|l|l|l|}
\hline$\alpha$ & $480 \mathrm{~kg} \cdot \mathrm{m}$ & $e$ & $1 \mathrm{~s}$ \\
\hline$a$ & $17.45 \mathrm{~kg} . \mathrm{m} / \mathrm{s}$ & $d$ & $2 \mathrm{~m}$ \\
\hline$b$ & $0.019 \mathrm{~kg}$ & $f$ & $0.02 \mathrm{~s}^{2} / \mathrm{m}$ \\
\hline$\tau$ & $0.05 \mathrm{~s}$ & $\bar{v}$ & $30 \mathrm{~m} / \mathrm{s}$ \\
\hline
\end{tabular}

Table 2: Numerical values for the parameters

Using the presented LMI conditions, the following gain matrices were obtained for the PDC control law:

$$
\begin{aligned}
& F_{1}=\left[\begin{array}{ll}
2.57 \cdot 10^{4} & 1.98
\end{array}\right], M_{1}=-1.052 \cdot 10^{5} \\
& F_{2}=\left[\begin{array}{ll}
2.67 \cdot 10^{4} & 2.15
\end{array}\right], M_{2}=-8.35 \cdot 10^{4} \\
& X=\left[\begin{array}{ccc}
3.07 \cdot 10^{-9} & -1.64 \cdot 10^{-5} & 3.70 \cdot 10^{-10} \\
-1.64 \cdot 10^{-5} & 0.18 & -8.15 \cdot 10^{-7} \\
3.70 \cdot 10^{-10} & -8.15 \cdot 10^{-7} & 6.95 \cdot 10^{-11}
\end{array}\right]
\end{aligned}
$$

The gains are quite important, this is due to the different signals scales $\left(10^{2}\right.$ for the control, $10^{-1}$ for the spacing error, $10^{1}$ for the speeds).

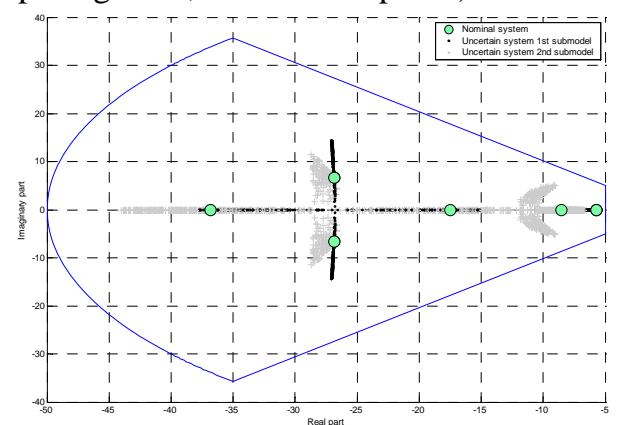

Fig. 4: closed-loop linear models poles location as a function of parameter's variations.

\subsection{Simulation results}

The initial conditions were $5 \mathrm{~m} / \mathrm{s}$ for both vehicles. The wheel torque was limited to $+600 \mathrm{Nm}$ and $-400 \mathrm{Nm}$. In order to illustrate the robustness of the proposed control, at time $t=60 \mathrm{~s}$, the controlled vehicle parameters are changed to $\alpha=721.19 \mathrm{~kg} . \mathrm{m}$, $a=26.18 \mathrm{~kg} . \mathrm{m} / \mathrm{s}$ and $b=0.01 \mathrm{~kg}$.

The leading vehicle speed is not realistic, but it has been chosen to emphasize the dynamics of the controlled vehicle. Both the leading and the following vehicle speeds are shown figure 5 .

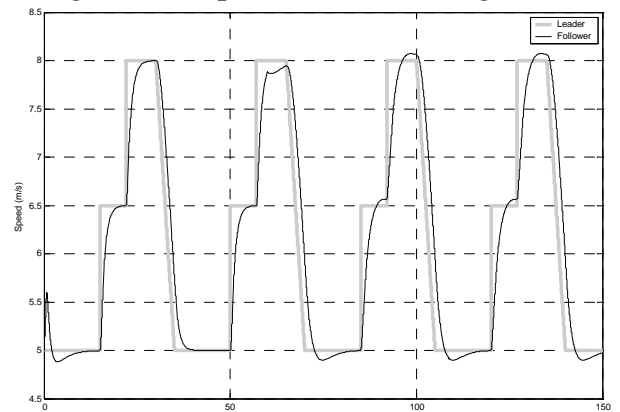

Fig. 5: Vehicles speed (simulation)

The spacing set point is a function of the following vehicle speed, figure 6. The initial tracking error is due to the initial conditions. During normal operation and despite the parameters changes, the spacing error does not exceed $5 \%$.
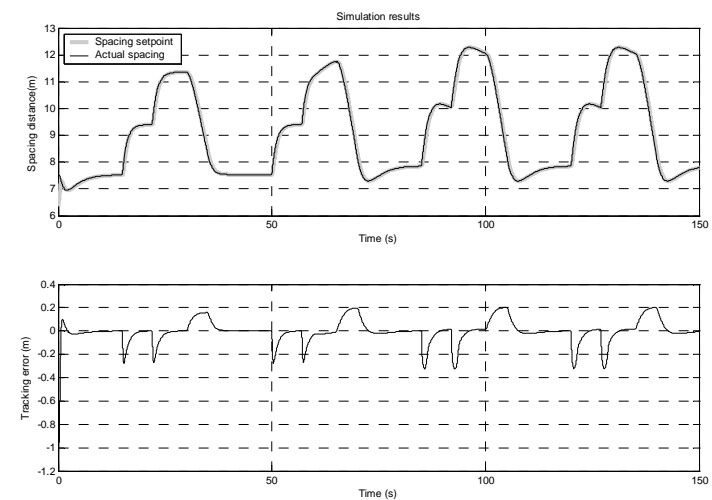

Fig. 6: Spacing error (simulation)

The wheel torque is represented figure 7 . The vehicle mass and aerodynamic drag being increased at time $t=60 \mathrm{~s}$, the torque required to ensure the same vehicle dynamic increases significantly.

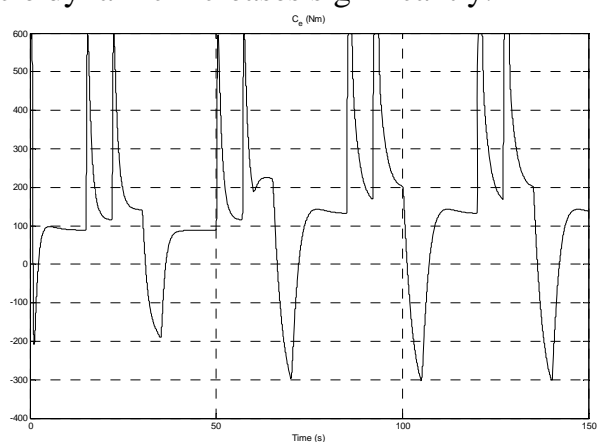

Fig. 7: Wheel torque (simulation)

\subsection{Real time experiments}

The experimental apparatus consists of one electric vehicle that follows a virtual leading vehicle. The spacing distance between the actual vehicle and the simulated one is computed by integrating the speed difference between both vehicles.

The following vehicle was driving on a straight lane until time $t=120 \mathrm{~s}$. At time $t=120 \mathrm{~s}$, the vehicle follows a traffic circle, and therefore important 
lateral effects that were not taken into account in the model occur.

The leading vehicle speed profile was similar to the simulation one, figure 8 .

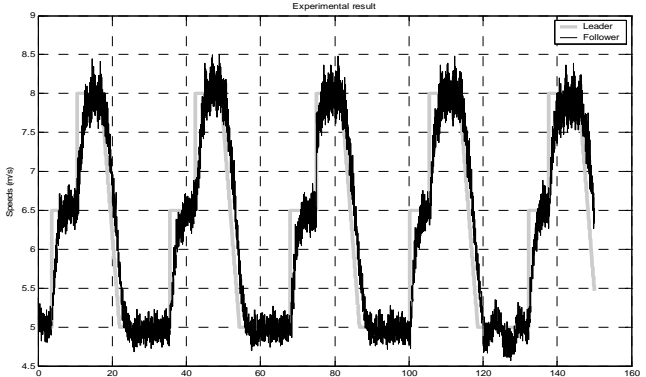

Fig. 8: Vehicle’s speeds (experimental result)

Real time results are quite similar with the simulation ones; the tracking error is less than $5 \%$ (figure 9).
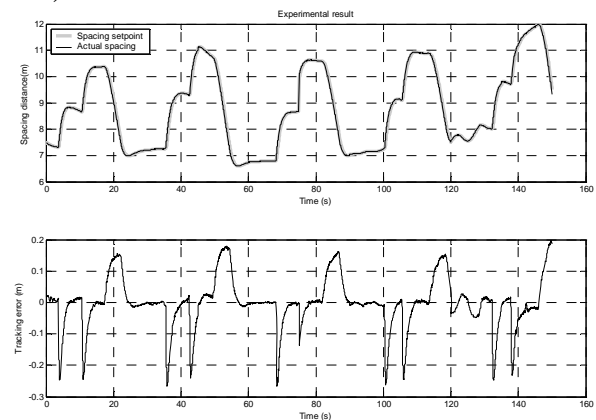

Fig. 9: Spacing error (experimental result)

The wheel torque was estimated using motor current sensing. The wheel torque evolution is quite similar to the simulation results. At time $t=120 \mathrm{~s}$, the traffic circle induced disturbances that were rejected.

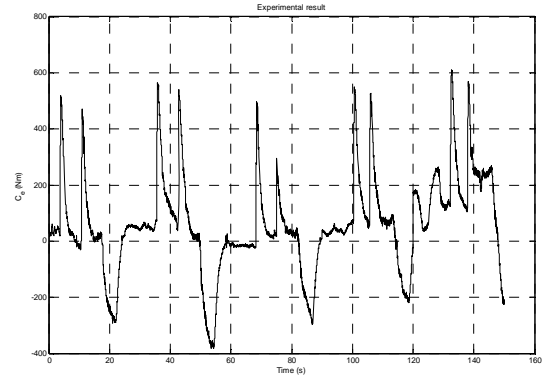

Fig. 10: Wheel torque (experimental result)

\section{Conclusion}

LMI conditions for robust pole placement in a LMI region for Takagi-Sugeno models were presented. Despite the fact that the closed loop dynamics can not be exactly specified by such a method, it is still a simple way to tune it.

The obtained conditions were applied to the spacing policy of an automated vehicle. Real time results were in accordance with simulation data.

On one hand, future work will be devoted to the extension of pole placement in a LMI region for PDC control with an observer and on the other hand, an experimental sensor will be tested in order to control both longitudinal and lateral dynamics with two real cars.

\section{REFERENCES}

Chilali M., Gahinet P., Apkarian P. (1999), Robust pole placement in LMI Regions, IEEE Trans.
Automatic Control, vol. 44, no. 12, p. 22572270, 1999.

Guerra T.M., Ksontini M. \& Delmotte F. (2003). Some new relaxed conditions of quadratic stabilization for continuous Takagi-Sugeno fuzzy models, IEEE CESA'03 Lille, France.

Guerra T.M., Vermeiren L. (2003), - Stabilité et stabilisation à partir de modèles flous - dans Commande floue 1 : «de la stabilisation à la supervision », sous la direction de L. Foulloy, S. Galichet \& A. Titli, IC2 Hermès, 59-98.

Hong S. L., Langari R. (2000), A LMI-based $H_{\infty}$ fuzzy control system design with TS framework, Information Sciences, 123 163-179.

Ioannou P (1994), Throttle and brake control systems for automatic vehicle following. IVHS J; 1(4): 345-377.

Lauber J., Guerra T.M., Perruquetti (2004), LMI conditions for continuous uncertain TS models in closed loop with an observer: application to engine speed control, Symposium IEEE-VTS Vehicle Power and Propulsion 2004, Paris, France.

Lee G.D. (2002), A longitudinal control system for a platoon of vehicles using a fuzzy-sliding mode algorithm, Mechatronics 12, pp. 97-118.

Liu X., Zhang O. (2003), New approaches to $H_{\infty}$ controller designs based on fuzzy observers for T-S fuzzy systems via LMI, Automatica, vol 39 (9), 1571-1582.

Nouvelière L. (2002), Commandes robustes appliquées au contrôle assisté d'un véhicule à basse vitesse, Thèse de Doctorat, Université de Versailles Saint Quentin en Yvelines.

Takagi T., Sugeno M. (1985), Fuzzy identification of systems and its application to modeling and control. IEEE Trans. on SMC, 15 (1) 116-132.

Tanaka, K., T. Ikeda, H.O. Wang (1998). Fuzzy regulators and fuzzy observers: relaxed stability conditions and LMI-based designs., IEEE Trans. on Fuzzy Systems, 6 (2), 1-16.

Taniguchi T., Tanaka K., Ohtake H., Wang H.O. (2001), Model construction, rule reduction and robust compensation for generalized form of Takagi-Sugeno fuzzy systems, IEEE Trans. on Fuzzy Systems, 9 (4), 525-537.

Tong S., Wang T., Li H.X. (2002), Fuzzy robust tracking control for uncertain non linear systems, International Journal of Approximate Reasoning, 30, 73-90.

Wang H.O., Tanaka K., Griffin M. (1996), An approach to fuzzy control of non linear systems: Stability and design issues, IEEE Transaction on Fuzzy Systems, 4 (1), 14-23.

Xie L., De Souza C.E. (1992), Robust $H_{\infty}$ control for linear systems with norm-bounded timevarying uncertainties, IEEE. Trans. On Automatic Control, 37, 1188-1191.

Zayed M., Rennuit A., Boonaert J. (2003), Plate Detection and Tracking for Automatic Car Following. International Crashworthiness and Design Symposium "Innovation Technologique Pour les Transports Terrestres", Lille (FRANCE). 